\title{
Aprender matemáticas en tiempos del COVID-19: Un estudio de caso con estudiantes universitarias
}

\section{Learning mathematics in times of COVID-19: A case study with university students}

\author{
Robinson Conde-Carmona \\ Universidad del Atlántico, Barranquilla, Colombia \\ Iván Padilla-Escorcia \\ Universidad del Norte, Barranquilla, Colombia
}

Open Access:

SSN: $0124-2121$

E-ISSN: $2665-2420$

\section{Resumen}

Objetivo: Analizar las percepciones de estudiantes universitarias sobre el aprendizaje de las matemáticas, específicamente de un curso de Cálculo Integral (Cálculo II), en el contexto de la pandemia por Covid-19 y el efecto que tuvo la transición de la presencialidad a la virtualidad. Método: Se realizó un estudio de caso con dos participantes, de contextos socioeconómicos y universidades diferentes. Se validaron los instrumentos mediante el método Delphi para mayor fiabilidad y confianza en los datos. Resultados: Se encontró que hubo discrepancias en la percepción de las participantes sobre el servicio prestado por las universidades. También hubo diferencias en la percepción de la forma como recibían las clases, el acceso a estas debido a la conectividad y a los equipos electrónicos con los que contaban. Para la estudiante 2, residente de un barrio tipificado como estrato cinco, el aprendizaje de cálculo integral en tiempos de pandemia, resultó un espacio para fomentar a la autonomía y auto estudio de una asignatura compleja como esta. En el caso de la estudiante 1, residente de un barrio tipificado como estrato uno, hubo la percepción de que el aprendizaje fue limitado, hubo desmotivación y críticas a la modalidad E-learning en el aprendizaje de asignaturas afines a la matemática. Discusión y Conclusiones: Se concluyó que, el estrato socioeconómico de las estudiantes y la disponibilidad de recursos tanto por parte de estas como de la universidad en donde estudian, influyeron en las percepciones sobre las clases virtuales.

Palabras clave: Covid-19, educación virtual, recursos virtuales, aprendizaje de la matemática, estudio de casos.

\begin{abstract}
Objective: To analyze the perceptions of college students about learning mathematics, specifically a course in integral Calculus (Calculus II) in the context of the Covid-19 pandemic, and the effect of the transition from face-to-face to online learning. Method: A case study was carried out with two participants from different socioeconomic contexts and universities. The instruments were validated using the Delphi method to ensure greater reliability and confidence in the data. Results: The study showed discrepancies in the perception that the participants had about the service provided by their universities. There were also differences in the perception of taking online lessons, of the access to these lessons due to the connectivity, as well as the availability of electronic equipment. For student 2, a resident of a neighborhood classified as stratum five, learning Integral Calculus in times of pandemic was a space provided to develop autonomy and self-study of a complex subject like this one. In the case of student 1, a resident of a neighborhood classified as stratum one, there was the perception that learning was limited, there was lack of motivation and criticism of the e-learning method for subjects related to mathematics. Discussion and Conclusion: It was concluded that the socioeconomic stratum of the students and the availability of their own resources, as well as the ones provided by their universities influenced their perceptions of virtual classes.
\end{abstract}

Keywords: Covid-19, virtual education, virtual resources, learning mathematics, case studies. 


\section{Introducción}

Con el pasar del tiempo, el uso de las nuevas tecnologías de la información y comunicación (TIC) han permitido flexibilidad en los procesos educativos. Asimismo, distintas modalidades han sido involucradas en la búsqueda de atender las dificultades y necesidades que en ocasiones suelen tener poblaciones que quieren acceder a la educación (Alvarado, Aragón y Bretones, 2020; Padilla-Escorcia y Conde-Carmona, 2020).

La educación virtual es una de las que mayor auge ha tomado como reemplazo de la educación tradicional y como estrategia para que cualquier persona, independientemente de su edad, pueda acceder (Francis, Wormington y Hulleman, 2019). Esta se concibe como una modalidad de fácil acceso, dado que se caracteriza porque la enseñanza de los contenidos es a distancia. Además, uno de sus pilares es ampliar la oportunidades de acceso a la educación y, por tal motivo, facilitar el ingreso de un mayor número de estudiantes que en ocasiones tienen inconvenientes de transporte y programación (Ministerio de Educación Nacional [MEN], 2010; Martínez, 2008; Martínez y García, 2020).

De acuerdo con Ibercampus (2015), la modalidad e-learning se hace cada vez más popular en los entornos académicos universitarios nacionales e internacionales. Se estima que, durante el año 2015 , el $50 \%$ de los universitarios de todo el mundo estaban inscritos en algún curso de esta modalidad y tres de cada cuatro universidades utilizaban las TIC en sus tareas académicas. Sin embargo, esta modalidad no solo depende del uso de la tecnología como mediadora de los procesos educativos, su éxito está definido en las relaciones que se establecen entre los sujetos que toman esta modalidad con el conocimiento mismo (Areth, Castro y Rodríguez, 2015). Esto es debido a que el nivel de la educación virtual varía según el enfoque que se adopte en cada centro educativo; se trata de un proceso complejo y desafiante en el diario vivir de cada uno de los actores que participan (Schoepp, 2005).

Los procesos de calidad de la modalidad virtual de enseñanza requieren la conexión de los diferentes actores (estudiantes, profesores) con los contenidos, actividades y tecnología, para promover el desarrollo óptimo de las competencias que satisfagan las expectativas de quienes estudian bajo esta modalidad ante la sociedad (Ruíz y Dávila, 2016).

No obstante, la educación virtual históricamente ha confrontado problemas que denotan dudas en sus procesos de calidad, específicamente en relación con la falta de planificación y control por parte del profesorado y las distintas estrategias que se ponen en marcha en los procesos de enseñanza-aprendizaje (Rodríguez y Niculcar, 2016). Por eso Hilmayr et al. (2020) aseguran que la enseñanza bajo estas condiciones no tiene un efecto en los resultados de aprendizaje, sino más bien son los factores contextuales como los diseños instruccionales que utiliza el profesorado y que se usan en esta modalidad los que garantizan 
buenos resultados en los educandos.

Siguiendo con la idea anterior, Wang et al. (2020) plantean que los ambientes virtuales de aprendizaje también son elementos influyentes en términos de la educación virtual ( $e$ learning) debido a que incluyen un rango significativo y mezcla de tecnologías que proporcionan una cantidad de funciones, características y posibilidades que se adaptan a los distintos estilos de aprendizaje de los educandos, por lo que esta modalidad se concibe más flexible con respecto a la educación presencial. Su éxito depende de cómo esta sea plasmada de acuerdo con las necesidades que presentan quienes deciden estudiar en esas condiciones.

En ese sentido, se hace evidente que a pesar de que el e-learning ha sido implementado desde hace varios años, su eficacia no está del todo determinada en los contextos educativos. Lo que es preocupante si se tiene en cuenta que, durante el año 2020, Colombia y el mundo en general, atraviesan crisis económicas, políticas, culturales, sociales y, por ende, educativas como consecuencia de la pandemia desatada por el Covid-19.

Ahora bien, teniendo en cuenta que esta enfermedad viral (causada por SARS-CoV-2) fue determinada con el rótulo de pandemia como se indicó, los centros educativos de la mayor parte del mundo fueron clausurados en su modalidad presencial. Colombia no fue la excepción y pese a las marcadas diferencias sociales que existen en el país, se propuso la modalidad e-learning en colegios y universidades públicas y privadas como medio para desarrollar saberes y competencias de acuerdo con el nivel académico. Esta decisión, discutible, fue acatada por los centros educativos.

Es importante tener presente que, de acuerdo con Zaharah y Kirilova (2020), las escuelas que tienen experiencia limitada o nula con e-learning presentarán máximas dificultades, especialmente si son los profesores los que no entienden cómo usar aplicaciones en línea tales como Zoom, Teams, Meet, Skype, Blackboard ultra, entre otras.

Ahora bien, en cuanto a la educación superior, es discutible pensar que el panorama de los estudiantes de una universidad privada es igual o similar a los de una universidad pública. Así las cosas, surgen ciertos interrogantes tales como: ¿cuentan los estudiantes de universidades públicas con recursos para recibir la enseñanza de los contenidos de las matemáticas mediante e-learning?, ¿la enseñanza de asignaturas afines a las matemáticas como Cálculo II mediante la modalidad e-learning repercutió en los procesos de aprendizaje de los estudiantes durante la pandemia del Covid-19?

Para dar respuestas a estas interrogantes, el objetivo de esta investigación es analizar las percepciones de estudiantes universitarias sobre el aprendizaje de las matemáticas, específicamente de un curso de Cálculo Integral (Cálculo II), en el contexto de la pandemia por Covid-19 y el efecto que tuvo la transición de la presencialidad a la virtualidad. 


\section{E-learning en Educación Matemática}

En relación con las matemáticas, han sido distintas las percepciones que se han tenido acerca de la modalidad e-learning en tiempos de pandemia para los procesos de aprendizaje.

En la investigación realizada por Sintema (2020), referente al efecto del Covid-19 en el rendimiento de los estudiantes en educación STEM (ciencia, tecnología, ingeniería y matemática), se obtuvo que las repercusiones de esta pandemia afectarán profundamente a Zambia, especialmente por la cantidad de horas presenciales de contacto que se perderán entre estudiantes - profesores; así mismo, por la escasez de recursos electrónicos en el país africano, que no está preparado para atender esta necesidad educativa mediante la modalidad e-learning, en especial para los estudiante de último grado que debían presentar pruebas nacionales en sus competencias STEM.

A través de encuestas realizadas a profesores en el estudio realizado por Sintema (2020), se evidenció que son pesimistas y consideran que habrá una caída en el porcentaje de aprobación de estudiantes de secundaria en los exámenes nacionales del 2020 si la epidemia de Covid-19 no está contenida lo antes posible. A esto debe agregarse que el calendario académico de la escuela en Zambia fue interrumpido con el año escolar apenas iniciado, con escasa preparación de los profesores en TIC y escasa dotación de recursos tecnológicos por parte de los estudiantes.

Lo anterior se relaciona también con la investigación realizada por Mailizar, Almanthari, Maulina y Bruce (2020) acerca de las percepciones de los estudiantes de secundaria y profesores acerca de los obstáculos, barreras y fortalezas de la implementación del elearning durante la pandemia del Covid-19 en Indonesia. En este se encontró que, en general, los profesores indonesios de matemática de los distintos niveles, educación básica, secundaria y superior, piensan que es un desafío el uso del e-learning y de herramientas tecnológicas para la enseñanza de esta disciplina durante la pandemia. La falta de conocimiento del estudiantado acerca del aprendizaje electrónico, sumado a la falta de acceso a este tipo de dispositivos y de conectividad a internet es una limitante.

Otro aporte significativo en este frente es la caracterización construida por Lubis et al. (2020), que contribuye a definir lo que se necesita para construir un módulo interactivo en modalidad e-learning, lo cual incluye facilidad de navegación, ya que un programa debe diseñarse lo más simple posible, puesto que además de contar con contenidos de tipo cognitivo, el contenido del programa debe proporcionar la experiencia que necesitan los estudiantes para aprender y complementada con la integración de las TIC. Además, debe integrar habilidades de lenguaje, escucha, habla, escritura, razonamiento y lectura. Todo esto para que cuando un estudiante termine de ejecutar un programa, sienta que ha aprendido algo.

Por su parte, en el contexto latinoamericano, Pochulu (2020) asegura que, en tiempos 
de pandemia del Covid-19, a los estudiantes en la enseñanza de las matemáticas se les debe modelizar situaciones internas y externas a esta disciplina, que involucren la construcción y análisis de modelos funcionales. Esto es pertinente dadas las circunstancias en el contexto latino y mundial con respecto a la modalidad e-learning para recibir clases.

\section{Metodología}

Esta investigación se desarrolla bajo los criterios del enfoque cualitativo, determinada por caracterizar la percepción, de elementos tales como, el acceso, la conectividad y el aprendizaje de las matemáticas en la modalidad virtual en tiempos del Covid-19 de dos estudiantes universitarias (Márquez y Olea, 2020). Además, utiliza la recolección de los datos para analizar y afinar las preguntas de la investigación, de manera que estas permitan revelar interrogantes surgidos en el proceso investigativo, esto se realizó en particular en las entrevistas, en las cuales, con ayuda de las participantes, además de refinar las preguntas investigativas, surgieron otras nuevas que permitieron orientar la investigación (Stake, 2010).

Su diseño es de estudio de caso ya que se basa en el análisis y descripción de fenómenos contemporáneos que se consideran prácticamente nuevos (Hancock y Algozzine 2006), y que en esta investigación corresponde al aprendizaje de las matemáticas por parte de dos estudiantes universitarias de estratos socioeconómicos distintos, en tiempos del Covid-19, mediante la modalidad e-learning.

Es un estudio de caso de tipo instrumental (Stake, 2005), ya que examina un caso particular, con el fin de proporcionar información sobre un tema o para formular una abstracción que, a los efectos de esta investigación, se refiere a la percepción sobre la modalidad e-learning en el aprendizaje de las matemáticas de dos estudiantes de instituciones universitarias, una de carácter público y otra de carácter privado. Así mismo, se indaga sobre la percepción que tienen de la formación de profesores en recursos digitales, la conectividad, los aparatos electrónicos, entre otros.

Para la selección de los casos, se utilizó el criterio de Simons (2011), con respecto a que en un estudio de caso instrumental, las y los participantes de la investigación pueden ser seleccionados de una serie de casos del tema que se esté trabajando en particular. Es así como la participante 1 y la participante 2 , se eligieron tomando en cuenta el hecho de que pertenecen a instituciones de carácter superior y de contextos diferentes, sumado a su interés por participar en la investigación, con su respectivo consentimiento informado.

Fases de la metodología adaptadas de Sintema (2020): 
1. Se realizaron entrevistas por medio de encuestas electrónicas y llamadas telefónicas por el aislamiento social de todos los ciudadanos debido a la pandemia.

2. Las entrevistas fueron grabadas, para transcribirlas y estudiarlas en detalle y por categorías.

3. Se usaron técnicas de análisis cualitativos, transcripción y codificación de la información, realizadas por los investigadores y por expertos externos en el tema de e-learning, para disipar sesgos. Las transcripciones se discutieron para evitar inconsistencias.

4. Se les pidió a las participantes que leyeran las transcripciones y confirmaran si estas representaban sus puntos de vistas. Esto hizo aumentar la validez y la fiabilidad de los datos.

\section{Participantes}

Criterios para la selección de las participantes: (i) estudiante de universidad pública o privada; (ii) ser estudiante de Ingeniería (iii); estar cursando alguna asignatura de matemática, para términos de homogeneidad, se escogió Cálculo Integral (Cálculo II) y, por último, (iv) contexto socioeconómico distintos, i.e., una participante en un estrato socioeconómico ${ }^{1}$ alto, otra participante en un estrato bajo.

Participante 1 (P1). Estudiante de tercer semestre de universidad pública, de 21 años de edad, perteneciente al programa de Ingeniería Mecatrónica. La universidad está ubicada en un municipio cercano a la ciudad de Barranquilla. De igual manera, la estudiante también es residente de un municipio cercano a Barranquilla, cuenta con una regular cobertura de internet y no cuenta con computador. Además, reside en un barrio tipificado como estrato uno

Participante 2 (P2). Estudiante de tercer semestre de universidad privada, de 20 años de edad, perteneciente al programa de Ingeniería Civil. La universidad está ubica en el norte de la ciudad de Barranquilla, residente en Barranquilla, cuenta con una excelente cobertura de internet, cuenta con dos computadores y una tableta. Reside en un barrio tipificado como estrato cinco.

\footnotetext{
${ }^{1}$ Según el Departamento Administrativo Nacional de Estadística (DANE) de Colombia: "La estratificación socioeconómica es una clasificación en estratos de los inmuebles residenciales que deben recibir servicios públicos. Se realiza principalmente para cobrar de manera diferencial por estratos los servicios públicos domiciliarios permitiendo asignar subsidios y cobrar contribuciones en esta área" (página web del DANE). Los estratos 1,2 y 3 corresponden a los usuarios con menos recursos que reciben subsidios; los estratos 5 y 6 son los que tienen mayores recursos económicos.
} 


\section{Instrumentos}

Se diseñó una entrevista con un cuestionario de 20 preguntas semiestructuradas, subdivididas en 5 categorías vinculadas con el objetivo de esta investigación: (i) proceso de aprendizaje de las matemáticas de las participantes durante el Covid-19, (ii) planificación de plataformas digitales para la enseñanza de Cálculo II, (iii) comprensión de los contenidos de manera virtual versus la modalidad presencial, (iv) motivación en el aprendizaje de Cálculo II mediante e-learning, (v) ventajas y desventajas de estudiar matemáticas a través de e-learning.

El cuestionario fue validado por el método Delphi, que es una técnica de deliberación sistemática en la que los investigadores recopilan opiniones de paneles de expertos. Esto permite a los expertos comunicarse anónimamente entre sí y luego los investigadores exploran la información subyacente recopilada sobre las ideas o problemas de enfoque (Linstone y Turoff, 2020; Brown y Casado, 1970; Cruz, 2009).

En este caso, los expertos son investigadores en educación matemática residentes en Europa y países de América Latina. Inicialmente estuvieron de acuerdo con las 5 categorías derivadas de las 20 preguntas propuestas por los investigadores. La versión final del instrumento se puso a prueba con otro estudiante de Ingeniería, para buscar la clara compresión de las preguntas, luego, no hubo necesidad de modificar preguntas de la versión final dado los resultados hallados como se muestra en la Tabla 1.

Tabla 1.

Aplicación final Delphi

\begin{tabular}{|c|c|c|c|c|c|c|c|c|c|}
\hline \multirow{7}{*}{$\begin{array}{l}\text { Factores que } \\
\text { agrupan a los } \\
\text { cinco } \\
\text { indicadores }\end{array}$} & \multirow[t]{2}{*}{$\mathbf{I}$} & \multicolumn{8}{|c|}{ Criterios } \\
\hline & & MA & BA & $\mathbf{A}$ & PA & I & Suma & $\begin{array}{l}\text { Promed } \\
\text { io }(\mathrm{pm})\end{array}$ & N-pm \\
\hline & 1 & 0,84 & 4,09 & 4,09 & 4,09 & 4,09 & 17,2 & 3,44 & $-0,448$ \\
\hline & 2 & 0,26 & 1,29 & 4,09 & 4,09 & 4,09 & 13,82 & 2,764 & 0,228 \\
\hline & 3 & 0 & 1,29 & 4,09 & 4,09 & 4,09 & 13,56 & 2,712 & 0,28 \\
\hline & 4 & 0,53 & 4,09 & 4,09 & 4,09 & 4,09 & 16,89 & 3,378 & $-0,386$ \\
\hline & 5 & 0 & 1,29 & 4,09 & 4,09 & 4,09 & 13,56 & 2,712 & 0,28 \\
\hline Suma & & 1,63 & 12,05 & 20,45 & 20,45 & 20,45 & 74,8 & 14,96 & \\
\hline Punto de corte & & 0,32 & 2,41 & 4,09 & 4,09 & 4,09 & 14,96 & & 2,992 \\
\hline $\begin{array}{l}\text { Promedios } \\
\text { promedios en } \mathbf{N}\end{array}$ & de & & & & & & & 2,992 & \\
\hline
\end{tabular}

Fuente: Elaboración propia

I: Indicadores. MA: Muy Adecuado. BA: Bastante Adecuado. A: Adecuado. PA: Poco Adecuado. I: Inadecuado.

A partir de la aplicación final de Delphi, los puntos de corte, determinados por el promedio de las columnas correspondientes a MB, BA, A, PA e I, se realizó la siguiente gráfica adaptada de Cruz (2009) (Figura 1). 
Figura 1.

Gráfico de los puntos de corte del método Delphi

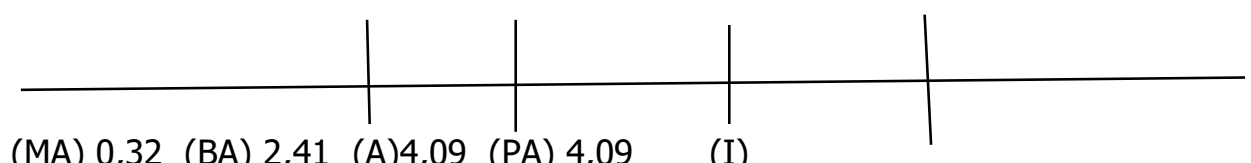

(MA) 0,32 (BA) 2,41 (A)4,09 (PA) 4,09

Fuente: Cruz (2009)

En esta se muestra claramente que en la última columna determinada con N-pm de la Tabla 1, que corresponde a la diferencia del promedio de los punto de corte con el promedio de las filas de cada categoría, todos los resultados se ubican en la categoría de MA, que está determinada por todos los valores menores a 0,32, incluyendo este, como se puede observar en la Figura 1, en la parte izquierda de la misma. Por ejemplo, el valor de la categoría 2 resultó ser 0,228 el cual es un valor menor a 0,32 y, por lo tanto, cumple con la condición antes mencionada. Es por esto que se denotó la validez y pertinencia en la aplicación de cada uno de las 5 categorías en las que se subdividieron las 20 preguntas de la entrevista aplicada a los 2 participantes de esta investigación. Se omitieron posibilidades y porcentaje de sesgo en los resultados, se obtuvieron datos confiables para futuros análisis.

\section{Resultados}

\section{Proceso de aprendizaje de las matemáticas de las participantes durante el Covid-19}

En esta categoría se ahondaron preguntas como: A partir de la contingencia ocasionada por el Covid-19, considera que esto ¿favoreció o no su proceso de aprendizaje?, ¿por qué? Resultó interesante observar que, a pesar de que las participantes vivencian el mismo proceso de aprendizaje desde casa mediante la modalidad e-learning, las respuestas dadas por cada una están condicionadas por sus estratos socioeconómicos.

La participante 1, perteneciente al estrato socioeconómico uno y sin una buena cobertura a internet, asegura que la pandemia desfavoreció en una proporción alta su proceso de aprendizaje, especialmente en la asignatura Cálculo II, cátedra en la que considera que es necesario la presencialidad para poder comprender cada uno de los procedimientos que conlleva el desarrollo de los distintos ejercicios y problemas. Manifestó, además que, en algunas ocasiones, no ha podido conectarse a las clases.

Esto contrasta con el estudio de Perienen (2020), quien asegura que, en las universidades de mayor prestigio en Zambia, existió mucho cuidado para que los estudiantes asistieran a 
las clases, inclusive los que no contaban con la mejor conectividad. Para esto las directrices institucionales estuvieron encaminadas en controlar que los estudiantes asistieran a las clases en medio de la pandemia utilizando Moodle y Astria como recursos en los procesos de aprendizaje.

Ahora bien, a diferencia de esto, la participante 2, de estrato socioeconómico alto, asegura que, por el contrario, la situación de contingencia ha permitido que los procesos de aprendizaje sean más autónomos debido a múltiples razones como la conectividad, como se observa en la siguiente afirmación textual que esta realiza a la pregunta mencionada anteriormente con respecto a si la pandemia favoreció o no su proceso de aprendizaje:

P2: Sí, ya que ha fomentado el autoestudio. Por las circunstancias, muchos estudiantes no pueden conectarse a una clase virtual, por lo que deben tomar la iniciativa de estudiar bajo lineamientos propios.

Es curioso que la P2, a pesar de tener los recursos como computadores y una conectividad estable en casa para recibir sus clases, considera que esto no es lo más relevante en los procesos de aprendizaje bajo la modalidad e-learning. Le da mayor importancia y relevancia a la propia capacidad del estudiante, mediante la lectura, vídeos de YouTube u otro tipo de estrategias, para aprender los contenidos que se enseñan en cada una de las asignaturas que cursan durante la contingencia.

En relación con Cálculo II, asignatura en común de las participantes de este estudio, P2 es clara con respecto a que, evidentemente, esta ha sido la asignatura que más dificultad le ha presentado durante este tiempo, dada su complejidad, por lo que considera clave en el éxito en el proceso de aprendizaje de esta, su propia capacidad de practicar y buscar por otros medios distintos a los que el profesor le explica en la modalidad e-learning.

En la investigación realizada por Longhurst et al. (2020) acerca de la educación virtual en el nivel universitario en tiempos del Covid-19 en el Reino Unido y la República de Irlanda, los resultados muestran que, en países con índice de desarrollo humano alto, se evidencian percepciones similares a las de la P2.

En el estudio mencionado en el párrafo precedente, se obtuvo que, el $71 \%$ de la población estudiantil destacó los recursos en línea, mientras que el 50\% de las universidades mencionan la colaboración académica como una oportunidad de fomento del aprendizaje cooperativo entre los pares (estudiantes). No obstante, cabe destacar que la mayor preocupación estuvo en el hecho de que se les da mayor importancia a las conferencias (con un porcentaje mayor al 50\%), con las que se pretende se pretenden reemplazar los aprendizajes prácticos en carreras como Ingeniería, lo que a su vez está relacionado con la poca participación de los estudiantes en este tipo de clases (Longhurst et al., 2020). 


\section{Planificación de plataformas digitales para la enseñanza de Cálculo II}

Las participantes son conscientes que en medio de la contingencia del Covid-19, la enseñanza de una asignatura como Cálculo II es difícil dada la complejidad de los contenidos; sin embargo, valoran que sus profesores planifiquen las clases y se esfuercen para que los contenidos que enseñan sean comprensibles. Para esto, utilizan recursos educativos digitales que favorecen la comprensión por parte de los estudiantes como tableros digitales, presentación de diapositivas en PowerPoint y plataformas como Zoom, Teams, Google Meet, Canvas, entre otros que permiten, a través de clases sincrónicas en su mayoría, y en algunas ocasiones asincrónicas, facilidad en la comunicación.

Debido al brote de Covid-19, los profesores se han visto en la necesidad de aprender métodos digitales de enseñanza y entrega de contenidos a sus estudiantes. Lo que ha provocado apreciar dispositivos digitales, recursos en línea, tecnología de redes sociales y actividades de aprendizaje electrónico (Mulenga, Marbán, 2020). Aparte de que, en tiempos de pandemia, múltiples académicos en Educación, se han solidarizado con los profesores y han ofrecido conferencias gratuitas a través de las redes sociales que más están al alcance de las personas (Facebook, Instagram, Twitter, entre otras), en donde ofrecen material educativo que potencia la enseñanza de sus contenidos bajo esta modalidad (Longhurst et al, 2020).

Ahora bien, es llamativo que a la pregunta ¿considera que su profesor(es) de Cálculo II cuenta con las competencias digitales para enseñar bajo estas condiciones de e-learning?, la cual fue realizada con el objetivo de que las participantes evaluaran el rol de sus profesores con las distintas plataformas digitales que utilizan durante la pandemia, ambas participantes resaltaron la labor del profesor, como textualmente se muestra a continuación:

P1: Sí, utiliza muy bien nuestra plataforma de clases.

P2: Sí, la utilización de herramientas como tableros digitales, vídeos, diapositivas, le han sido de gran ayuda para el desarrollo de la clase, permitiéndole al estudiante comprender eficientemente los temas tratados en la asignatura.

En contraste con la $\mathrm{P} 1$, la $\mathrm{P} 2$ resalta aún más a su profesor en tiempos de pandemia, lo que en parte, puede obedecer a que la P2 cuenta con mejor conexión de internet desde casa y por lo tanto puede acceder a observar las clases de su profesor de manera rutinaria y sin ningún inconveniente.

No obstante, en el caso de la P1, tiene mayores obstáculos y dificultades en la obtención de los conocimientos, no tanto porque sus profesores no hayan sabido manejar la situación durante estos tiempos del Covid-19, sino a las dificultades vinculadas con internet en su casa, como se indicó, lo que ha sido una limitante para el acceso a clases de calidad mediante la modalidad e-learning. 


\section{Comprensión de los contenidos de manera virtual versus modalidad presencial}

En esta categoría se abordaron preguntas como las siguientes:

- Desde su percepción, ¿̇la comprensión que ha tenido de los contenidos de matemáticas a través de la implementación de la enseñanza virtual en su centro de estudios ha sido similar o diferente a la comprensión que tenía de manera presencial?

- Explique, de acuerdo con su experiencia, ¿bajo qué modalidad, virtual o presencial, se siente más a gusto en el aprendizaje de las matemáticas?

La P2 resaltó una vez más que en la modalidad virtual ha comprendido en su totalidad los contenidos, particularmente los de la asignatura Cálculo II, como si estuviera en modalidad presencial, ya que su profesor ha buscado todos los recursos digitales posibles para facilitar el aprendizaje en los estudiantes. Pese a esto, la P2 tiene mayor preferencia por la presencialidad, como se observa en su respuesta.

P2: Presencial, puedo sentirme cómoda con la modalidad virtual por comprender la pandemia que atraviesa el mundo, pero presencial es sin duda la modalidad en la que me siento a gusto porque no hay nada como exponer tus dudas y que el docente pueda resolverlas viendo el proceso presencial del estudiante.

La P2 entiende, comprende y acepta la situación de contingencia para aprender de manera virtual. De acuerdo con Perienen (2020), en el caso de las matemáticas, este método de e-learning es apropiado solamente cuando los estudiantes aprenden de manera efectiva. Sin embargo, se considera que esta modalidad no cuenta con algo muy valioso en el proceso de aprendizaje de los estudiantes y es que el profesor acompañe el proceso y verifique que realmente los resultados de aprendizaje esperados se estén logrando de manera eficiente.

Cabe señalar que la respuesta dada por la P2, es de las pocas en las que coincidió con la $\mathrm{P} 1$, quien piensa igual con respecto a que de manera presencial es la única existente en la que un estudiante puede aclarar las dudas que tiene con respecto a determinada asignatura, más si esta trata de números, como en la cátedra de Cálculo II.

Por otra parte, P1 a su vez considera que la modalidad virtual limita al profesor para que clarifique y ejemplifique las dudas que los estudiantes tienen, como expresa en uno de sus comentarios, donde denota su preferencia por la modalidad presencial.

P1: Mi proceso de aprendizaje en la modalidad virtual ha sido diferente, ya que de manera presencial podía acercarme personalmente a donde mi profesor y pedir que me aclarara alguna duda y me la aclaraba con un ejemplo en el tablero.

De acuerdo con lo manifestado por P1, es probable que el profesor de Cálculo II no utilice 
tabletas digitalizadoras o WiziQ, los cuales son conocidos como tableros virtuales y que, según Parra (2020), permiten mayor interacción entre los profesores y estudiantes y por tal motivo, estos últimos se sienten como si estuvieran en clases de manera presencial.

\section{Motivación al aprendizaje de Cálculo II mediante el e-learning}

En esta categoría se confirmó una vez más la diferencia de percepciones entre la P1 y la P2 en la situación de contingencia, cuyo desnivel está enmarcado en la favorabilidad que tiene P2 para poder recibir sus clases de manera sincrónica mediante la modalidad $e$ learning.

En el caso de la P1, las dificultades de conexión y otras razones, generan en ella desmotivación en el aprendizaje del Cálculo II en época de pandemia, como se evidencia en la respuesta que da a la pregunta: ¿cómo considera su motivación hacia el aprendizaje de Cálculo II a través de la modalidad virtual con respecto a la modalidad presencial?

La P1 expresa que no existe motivación para trabajar las clases de Cálculo II de manera virtual, dado que no se aclaran las dudas, en algunas ocasiones la conexión es limitada y además, debido a que las matemáticas, al ser esta una ciencia compleja, requiere de explicaciones claras, precisas y concisas.

Piensa la P1 que su motivación no es la misma desde que siente que no está preparada para realizar un examen o taller de la misma forma que cuando estaba de manera presencial en el salón de clases. Afirma que su seguridad disminuyó debido a que la enseñanza mediante e-learning es muy desventajosa para los estudiantes.

Esto a su vez muestra que el e-learning genera dilemas para los estudiantes en tiempos de pandemia, pero también para los profesores, orientados en la triada profesor-estudianteaprendizaje. Dado que este modelo provoca disrupciones pedagógicas y transformaciones en las interacciones que solían tener los estudiantes con el ambiente de aprendizaje en la modalidad presencial, debido al método de enseñanza del profesor y a las variaciones en las organizaciones curriculares, lo que es causa de desinterés en el proceso de aprendizaje (Parra, 2020).

La respuesta que da la $\mathrm{P} 2$, con respecto a si se siente motivada y preparada para realizar un examen de Cálculo II a partir de la inserción del e-learning en las clases, es diferente a la de la P1, como se observa a continuación:

P2: Me siento igual de preparada, porque el aprendizaje no depende únicamente del docente, sino del estudiante, este es quien decide aumentarlo o hacer parte de la mediocridad.

Esta respuesta es muy distinta a la de la P1 y coherente con las ideas que la P2 manifestó durante las entrevistas realizadas: que la modalidad e-learning es únicamente el medio para 
facilitar el aprendizaje, pero es el estudiante quien decide si es efectivo o no, por lo que se apreciar que la motivación de P2 está determinada por su propio interés para querer aprender, en este caso, Cálculo II.

\section{Ventajas y desventajas de estudiar matemáticas a través del e-learning}

Ante la pregunta: - ¿Considera como ventajoso o desventajoso la metodología de enseñanza virtual (e-learning) de los contenidos de matemáticas? Explique, las opiniones de las participantes fueron las siguientes.

La P1 es contundente en que es solamente desventajoso ya que los contenidos no son totalmente comprendidos por los estudiantes. Asegura que la universidad de la cual es estudiante, no se adaptó a dicha metodología (e-learning), dado que muchos estudiantes de su condición no pueden recibir clases con calidad, puesto que su cobertura a internet no es la mejor, por lo que considera lo siguiente:

P1: La metodología e-learning, a mi punto de vista, es más desventajosa que ventajosa, ya que hay menos comprensión de los temas, y los estudiantes no siguen un proceso, y los profesores, a pesar de que se esfuerzan, no pueden atender en su totalidad las dudas que tenemos.

Es llamativo que a pesar que la modalidad e-learning es flexible en cuanto a la entrega de tareas, los encuentros en los cuales un estudiante no puede asistir de manera remota pueden ser vistos en otro horario, la P1 no contempla nada de lo anterior, específicamente porque bien sea de manera sincrónica o asincrónica, su internet no le permite acceder a los mismos de buena forma.

Por su parte, la P2 es más optimista en pensar que estudiar bajo la metodología elearning es ventajoso, ya que promueve el autoestudio y además les permite a los estudiantes darse cuenta que los contenidos de una asignatura compleja como Cálculo II pueden ser aprendidos en diferentes modalidades y tiempos y sin depender al $100 \%$ de lo que el profesor explica en clase. Afirmó que utiliza otras herramientas para aprender y consolidar sus conocimientos:

P2: A través de videos y libros, logro desarrollar positivamente mi aprendizaje de las matemáticas.

Asimismo, afirma la P2 que resulta ventajoso poder organizar un horario de trabajo más flexible bajo esta modalidad y además, las clases que los profesores explican de manera remota pueden ser observadas posteriormente, lo que muchas veces es indispensable para aclarar dudas e inquietudes. 


\section{Discusiones}

La contingencia ocasionada por el Covid-19 aún mantiene suspendidas las clases presenciales en muchas partes del mundo. América Latina no es la excepción, a pesar de que la pandemia tardó en llegar a esta región.

La modalidad e-learning se ha convertido en el método más efectivo para que los estudiantes en cada uno de los niveles de escolaridad atiendan sus clases.

No obstante, en el contexto universitario, llevar a cabo las clases de manera efectiva ha generado mayores dificultades, dado que son muchas las carreras que requieren la práctica como elemento clave en los procesos de aprendizaje de los educandos. Es así como esta investigación presenta contrastes con estudios en países con economía similares a las de Colombia y países que cuentan con mejores recursos. Las investigaciones realizadas por Longhurst et al., 2020; Mailizar et al., (2020) y Sintema (2020) son evidencias de ello. En estas se percibe que, en países en vía de desarrollo como Indonesia y Zambia, los estudiantes presentan dificultades para conectarse a muchas de las clases bajo la modalidad e-learning por limitaciones en la cobertura de conectividad y escasez de recursos tecnológicos. Lo cual es semejante a lo afirmado por la P1 de esta investigación. Estas razones provocan desmotivación y deserción escolar en los estudiantes.

Lo descrito anteriormente es diferente a lo que ocurre en Reino Unido y la República de Irlanda, donde la percepción de los estudiantes acerca de la modalidad virtual en tiempos de pandemia es favorable en más del $50 \%$. Esto muestra que, contar con los recursos y una buena conexión a internet, aumenta el interés por los procesos de aprendizaje. Estos resultados se asemejan a los mostrados en la percepción de la P2 de esta investigación, quien en la mayoría de los casos, no presenta quejas acerca de la forma en que su universidad y profesores han asumido la enseñanza de los contenidos bajo la coyuntura del Covid-19.

Sin embargo, Cortez (2020) afirma en sus resultados de investigación, que la capacidad de los estudiantes para asistir a sus clases mediante e-learning es independiente de su estilo de vida, lo cual está muy relacionado con que los programas prácticos se han visto desfavorecidos, ya que no es suficiente una conferencia para cubrir las necesidades de carreras como Ingeniería (Longhurst, 2020), programa al cual pertenecen las participantes de este estudio.

Es importante destacar que, la P1 mencionó en el cuestionario que probablemente el próximo semestre no lo estudiará debido a razones de conexión, pero sobre todo de tipo práctico, ya que no le encuentra al sentido a estudiar sin encontrar la aplicación de lo que estudia, como fue muy notorio en su curso de Cálculo II, asignatura con mayor dificultad para ambas participantes en este semestre.

Para culminar este punto, es importante hacer un contraste entre las respuestas de las 
estudiantes de este estudio con la investigación realizada por Parra et al. (2016), con respecto a la deserción estudiantil en una universidad colombiana. Este estudio encontró que la deserción en los programas en modalidad virtual en promedio fue de $76 \%$, mientras que en los de modalidad presencial fue de $51 \%$ (similar a las cifras nacionales en ingeniería). Este dato es preocupante, si se tiene en cuenta que hace 4 años no estaba la pandemia. Lo que refleja que, en el ámbito de la ingeniería, se requiere estar alertas y evaluar la importancia de la presencialidad para llevar a cabo las prácticas.

\section{Conclusión}

Entre los hallazgos relevantes se tiene que las diferencias socioeconómicas y de recursos disponibles de las universidades donde estudian la P1 y la P2 fue determinante en la motivación de cada una de estas durante la enseñanza de Cálculo II en tiempos del Covid19.

Existen diferencias significativas y contrastes en la percepción de cada una de ellas. En el caso de la P1, esta debió enfrentar mayores dificultades para recibir sus clases, debido a que no contaba con la mejor conectividad y equipo de trabajo en casa. Esto incidió en la calidad del servicio que recibía, además que la universidad donde estudia, no cuenta con recursos suficientes para atender la diversidad de estudiantes sin conexión a internet o a aparatos electrónicos que les permitan desarrollar las clases de manera sincrónica.

A diferencia de esto, la P2, por su parte, consideró motivante y ventajoso poder aprender bajo la modalidad e-learning, dado que era una oportunidad para fomentar la autonomía y el autoestudio, y más aún en asignaturas con nivel de complejidad alto como Cálculo II, donde no es suficiente desarrollar aprendizaje solamente con lo que el profesor explica en clase.

Lo anterior invita a una posible proyección de este estudio en el mismo contexto, en una segunda etapa, en donde se tomen un mayor número de participantes de la universidad pública en búsqueda de identificar de los diferentes contrastes de esta población.

Además, es importante mencionar el papel fundamental que ha tenido la educación virtual, dadas las dificultades ocasionadas por la pandemia Covid-19, lo que ha permitido la continuidad de los procesos académicos en las universidades e incluso, se ha intentado mantener los horarios de clase presenciales y en muchos casos, se ha preservado la calidad del servicio.

No obstante, es importante tener presente que, para una asignatura con un alto componente práctico como Cálculo II, dicha calidad se ha visto afectada significativamente en el caso de estudiantes con dificultades de conexión y carencia de aparatos electrónicos para recibir las clases, como en el caso de la P1. 
Cabe destacar, además, la percepción que tuvieron ambas participantes en relación con la labor de los profesores y sus habilidades adaptativas, debido a que les tocó cambiar de modalidad, estrategias y metodologías de un día a otro, sin que esto afectara profundamente su calidad como docentes.

\section{Recomendaciones}

Para finalizar, se recomienda que, dentro de los recursos a utilizar por parte del profesorado de matemáticas en la modalidad e-learning se encuentren tabletas digitalizadoras o WiziQ, las cuales permiten interacción entre los profesores y estudiantes, ya que se asemejan a la presencialidad y permiten que los estudiantes resuelven ejercicios en la pizarra. También la utilización de plataformas de videoconferencias como Google Meet o Blackboard ultra, entre otras, que están sincronizadas con los correos electrónicos y que dada su buena resolución para compartir pantalla, permiten explicar la teoría de cada uno de los contenidos. Además, los videos grabados por el profesor son de gran ayuda para la solución de problemas, ya que permiten que este aborde situaciones con un nivel de complejidad mayor y que puedan ser analizados e interpretados por los estudiantes.

\section{Referencias}

Alvarado, L., Aragón R., \& Bretones F. (2020). Teachers' Attitudes Towards the Introduction of ICT in Ecuadorian Public Schools. TechTrends, (64), 498-505. https://doi.org/10.1007/s11528-020-00483-7

Areth J., Castro-Martínez, J., y Rodríguez, H. (2015). La educación virtual en Colombia: exposición de modelos de deserción. Revista apertura, 7(1), 1-10

Brown, B., y Casado, M. (1970). La Técnica Delfos: Metodología usada para obtener la opinión de los expertos. Revista española de la opinión pública, (21), 217-226. https://doi.org/10.2307/40181465

Cortez, C. (2020). Blended, Distance, Electronic and Virtual-Learning for the New Normal of Mathematics Education: A Senior High School Student's Perception. European Journal of Interactive Multimedia and Education, 1(1), 1-6. https://doi.org/10.30935/ejimed/8276

Cruz, M. (2009). El método Delphi en las investigaciones educacionales. Editorial Academia.

Departamento Administrativo Nacional de Estadística-DANE. (2020). Estratificación socioeconómica para servicios dúblicos domiciliarios.

https://www.dane.gov.co/index.php/servicios-al-ciudadano/serviciosinformacion/estratificacion-socioeconomica 
Francis, M., Wormington, S., \& Hulleman, C. (2019). The Cost of Online Learning: Examining Differences in Motivation and Academic Outcomes in Online and Face-to-Face Community College Developmental Mathematics Courses. Frontiers in Psychology, (10), 1-12. https://doi.org/10.3389/fpsyg.2019.02054

Hancock, D., \& Algozzine, B. (2006). Doing Case Study Research. Teachers College Columbia University.

Hillmayr, D., Ziernwald, L., Reinhold, F., \& Hofer, S. (2020). The potential of digital tools to enhance mathematics and science learning in secondary schools: A context-specific meta-analysis. Computers \& Education, (153), 1-25. https://doi.org/10.1016/j.compuedu.2020.103897

Ibercampus. (2015). El 50\% de los universitarios de todo el mundo están inscritos en cursos de e-Learning. http://www.ibercampus.es/articulo.asp?idarticulo=30744

Linstone, H., \& Turrof, M. (2020). The Delphi Method: Techniques and Applications. University of Southern California.

Longhurst, G., Stone, D., Dulohery, K., Scully, D., Campbell, D., \& Smith, C. (2020). Strength, Weakness, Opportunity, Threat (SWOT) Analysis of the Adaptations to Anatomical Education in the United Kingdom and Republic of Ireland in Response to the Covid-19 Pandemic. Anatomical Science Education, 13(3) 301-311. https://doi.org/10.1002/ase.1967

Lubis, A., Ritonga, A., Hia, Y., \& Nasution, A. A. (2020). Online Learning Design At Higher Education: An Example From Mathematics Classroom. JPhCS, 1462(1), 1-20.

Mailizar., Almanthari, A., Maulina, S., \& Bruce, S. (2020). Secondary School Mathematics Teachers' Views on E-learning Implementation Barriers during the Covid-19 Pandemic: The case of Indonesia. EURASIA Journal of Mathematics, Science and Technology Education, 16(7), 1-9. https://doi.org/10.29333/ejmste/8240

Márquez-Mosquera, V. A., \& Olea-Isaza, I. C. (2020). Las Actividades Orientadoras de Enseñanza como estrategia para enseñar la probabilidad en primaria: reflexiones de los maestros. Trilogía Ciencia Tecnología Sociedad, 12(22), 151-171. https://doi.org/10.22430/21457778.1094

Martínez. J. (2008). La educación a distancia: sus características y necesidad en la educación actual. Educación, 17(33), 7-27.

Martínez, J., y Garcés, J. (2020). Competencias digitales docentes y el reto de la educación virtual derivado de la Covid-19. Educación y Humanismo, 22(39), 1-16. https://doi.org/10.17081/eduhum.22.39.4114 
MEN. (2010). Deserción estudiantil en la educación superior colombiana: metodología de seguimiento, diagnóstico y elementos para su prevención. Ministerio de Educación Nacional.

Mulenga, E., \& Marbán, J. (2020). Is COVID-19 the Gateway for Digital Learning in Mathematics Education? ContemporaryEducationalTechnology, 12(2), 1-11.

\section{https://doi.org/10.30935/cedtech/7949}

Padilla-Escorcia, I., \& Conde-Carmona, R. (2020). Uso y formación en TIC en profesores de matemáticas. Revista Virtual Universidad Católica del Norte, (60), 116-136. https://www.doi.org/10.35575/rvucn.n60a7

Parra, J. (2020). Prácticas de docencia tradicional en ambientes de educación virtual. Revista Academia y virtualidad, 13 (1), 93 -106. DOI: https://doi.org/10.18359/ravi.4295

Parra, C., Castañeda, E., Restrepo, G., Úsuga, O., Duque, P., \& Mendoza, R. (2016). ¿La deserción y la graduación no diferencian a los programas de pregrado de la Facultad de Ingeniería de la Universidad de Antioquía? En D. Gómez (comp.). IV Conferencia Latinoamericana sobre Abandono de la Educación Superior, 71-82. Medellín.

Perienen, A. (2020). Frameworks for ICT Integration in Mathematics Education - A Teacher's Perspective. Eurasia Journal of Mathematics, Science and Technology Education, 16(6), 1-12. https://doi.org/10.29333/ejmste/7803

Pochulu, M (2020). Pensar la enseñanza de las matemáticas en la virtualidad en tiempo récord Cuevas, conexión moral en la intimidación escolar [conferencia]. Conferencia presentada en Ciclo de conferencias en Educación Matemática de Gemad, Bogotá, Colombia. http://funes.uniandes.edu.co/17228/1/Pochulu2020Pensar.pdf

Rodríguez, D., y Niculcar, E. (2016). Las buenas prácticas docentes en la educación virtual universitaria. Revista de docencia universitaria: REDU, 14(2), 159-186.

Ruíz, C., \& Dávila. A. (2016). Propuesta de buenas prácticas de educación virtual en el contexto universitario. Revista de Educación a Distancia, 49(12), 1-21. http://dx.doi.org/10.6018/red/49/

Schoepp, K. (2005). Barriers to technology integration in a technology-rich environment. Learning and Teaching in Higher Education: Gulf Perspectives, 2(1), 1-24.

Simons, H. (2011). El estudio de caso: Teoría y práctica. Ediciones Morata, S.L.

Sintema, E. (2020). Effect of COVID-19 on the Performance of Grade 12 Students: Implications for STEM Education. EURASIA Journal of Mathematics, Science and Technology Education, 16(7), 1-6. https://doi.org/10.29333/ejmste/7893 
Stake, R. (2010). Qualitative research. Studying how things work. The Gilford Press.

Stake, R. (2005). Qualitative Case Studies. In N. K. Denzin \& Y. S. Lincoln (Eds.), The Sage handbook of qualitative research, $443-466$.

Wang, R., Lowe, R., Newton, S., \& Kocaturk, T. (2020). Task complexity and learning styles in situated virtual learning environments for construction higher education. Automation in Construction, (113), 1-11, https://doi.org/10.1016/j.autcon.2020.103148

Zaharah, Z., \& Kirilova, G. (2020). Impact of Corona Virus Outbreak Towards Teaching and Learning Activities in Indonesia. SALAM: JurnalSosial dan BudayaSyar-i, 7(3), 269282. http://journal.uinjkt.ac.id/index.php/salam/article/view/15104/0 